\title{
Lack of efficacy of an intradural somatic-to-autonomic nerve anastomosis (Xiao procedure) for bladder control in children with myelomeningocele and lipomyelomeningocele: results of a prospective, randomized, double-blind study
}

\author{
Gerald F. Tuite, MD, ${ }^{1,4,5}$ Ethan G. Polsky, MD, ${ }^{7}$ Yves Homsy, MD, ${ }^{7}$ Margaret A. Reilly, PT, ${ }^{2}$ \\ Carolyn M. Carey, MD, MBA, ${ }^{1,5}$ S. Parrish Winesett, MD, ${ }^{6}$ Luis F. Rodriguez, MD, ${ }^{1,5}$ \\ Bruce B. Storrs, MD, ${ }^{1,5}$ Sarah J. Gaskill, MD, ${ }^{5}$ Lisa L. Tetreault, RN, CCRP, ${ }^{3}$ \\ Denise G. Martinez, MPH, ${ }^{3}$ and Ernest K. Amankwah, $\mathrm{PhD}^{3}$
}

\begin{abstract}
${ }^{1}$ Division of Pediatric Neurosurgery, Neuroscience Institute, ${ }^{2}$ Department of Occupational and Physical Therapy, and ${ }^{3} \mathrm{Clinical}$ and Translational Research Organization, Johns Hopkins All Children's Hospital, St. Petersburg, Florida; ${ }^{4}$ Department of Pediatrics, Johns Hopkins Medicine, Baltimore, Maryland; ${ }^{2}$ Division of Pediatric Neurosurgery, Department of Neurosurgery and Brain Repair, and ${ }^{6}$ Division of Pediatric Neurology, Department of Pediatrics, University of South Florida, Tampa; and ${ }^{7}$ Children's Urology Group, Tampa, Florida
\end{abstract}

\begin{abstract}
OBJECTIVE Xiao et al. and other investigators have studied an intradural somatic-to-autonomic (e.g., L-5 to S3-4) nerve transfer as a method to create a reflex arc to allow bladder emptying in response to cutaneous stimulation (the Xiao procedure). In previous clinical studies of patients with spinal dysraphism who underwent the Xiao procedure, high success rates (70\%-85\%) were reported for the establishment of a "skin-CNS-bladder" reflex arc that allows spontaneous, controlled voiding in children with neurogenic bladder dysfunction. However, many of these studies did not use blinded observers, did not have control groups, and/or featured only limited follow-up durations.
\end{abstract}

METHODS A randomized, prospective, double-blind trial was initiated in March 2009, enrolling children with myelomeningocele (MM), lipomyelomeningocele (LMM), and neurogenic bladder dysfunction who were scheduled for spinal cord detethering (DT) for the usual indications. At the time of DT, patients were randomized between 2 arms of the study: half of the patients underwent a standard spinal cord DT procedure alone (DT group) and half underwent DT as well as the Xiao procedure (DT+X group). Patients, families, and study investigators, all of whom were blinded to the surgical details, analyzed the patients' strength, sensory function, mobility, voiding, and urodynamic bladder function before surgery and at regular intervals during the 3-year follow-up.

RESULTS Twenty patients were enrolled in the study: 10 underwent only DT and the other 10 underwent DT+X. The addition of the Xiao procedure to spinal cord DT resulted in longer operative times $(p=0.024)$ and a greater chance of wound infection $(p=0.03)$. Patients in both treatment arms could intermittently void or dribble small amounts of urine $(<20 \%$ total bladder capacity) in response to scratching in dermatomes T-9 through S-2 using a standardized protocol, but the voiding was not reproducible and the volume voided was not clinically useful in any patient. Voiding in response to scratching was not more frequent in patients who underwent $D T+X$ compared with those who underwent only DT. Bladder contractions in response to scratching occurred in both treatment arms at various intervals after surgery, but they were not more reproducible or more frequent in the patients who underwent the Xiao procedure than in the patients who did not. No patient in either treatment arm was continent of urine before, during, or after the study.

CONCLUSIONS Patients with MM and LMM who underwent the Xiao procedure during spinal cord DT were no more likely to be able to void, to control their urination, to achieve continence, or to have a demonstrable urodynamic bladder

ABBREVIATIONS BAM = bladder-active medication; $\mathrm{CIC}=$ clean intermittent catheterization; $\mathrm{DSMB}=$ data safety and monitoring board; $\mathrm{DT}=$ detethering; $\mathrm{EMG}=$ electromyography; LMM = lipomyelomeningocele; $\mathrm{MM}=$ myelomeningocele; $\mathrm{MMT}=$ manual muscle testing; PEDI = Pediatric Evaluation of Disability Inventory; QOL = quality of life; $\mathrm{TBC}=$ total bladder capacity; $\mathrm{X}=$ Xiao procedure.

ACCOMPANYING EDITORIALS AND RESPONSE See pp 145-149. DOls: 10.3171/2015.11.PEDS15623; 10.3171/2015.12.PEDS15633; and 10.3171/2015.12.PEDS15710. SUBMITTED May 1, 2015. ACCEPTED October 21, 2015.

INCLUDE WHEN CITING Published online May 3, 2016; DOI: 10.3171/2015.10.PEDS15271. 
contraction in response to cutaneous stimulation than patients who underwent only spinal cord DT. This study, in the context of disappointing results reported in other recent studies of the Xiao procedure, raises doubts about the clinical applicability of this procedure in humans until further basic science research is performed.

http://thejns.org/doi/abs/10.3171/2015.10.PEDS15271

KEY WORDS spina bifida; tethered spinal cord; bladder; Xiao procedure; nerve transfer; voiding; spinal dysraphism; congenital

$\mathrm{N}$ EUROGENIC bladder dysfunction related to spinal dysraphism leads to urinary incontinence and an inability to empty the bladder, with a propensity for urinary tract infections and renal dysfunction. ${ }^{5}$ Clean intermittent catheterization (CIC), the use of bladder-active medications (BAMs), and close urological follow-up can mitigate many of the potentially life-threatening complications of neurogenic bladder dysfunction, but patients' quality of life (QOL) is considerably affected by the lifelong burden of these interventions, the lack of control of their ability to empty their bladders, and by incontinence and sexual dysfunction. . $^{4,9,10,13,15,21,25,28-30}$

For more than 100 years, researchers and clinicians have pursued a myriad of surgical remedies to allow reinnervation of the urinary bladder, attempting a variety of nerve repair and transfer techniques in experimental animals and in humans. ${ }^{11}$ While there has been great success in the clinical use of nerve transfer techniques to reinnervate peripheral nerves of the extremities, ${ }^{3}$ there is no surgical nerve transfer or repair procedure that is widely used in patients with neurogenic bladder dysfunction. ${ }^{11}$

A recent review outlines many of the surgical techniques that have been pursued, ${ }^{11}$ including the "skinCNS-bladder" reflex pathway that was developed by Xiao et al. in a rat model more than 20 years ago. ${ }^{37}$ The Xiao procedure aims to establish an "artificial somatic-central nervous system-autonomic reflex" pathway for controlled micturition by modifying spinal cord-mediated reflex arcs, which may be preserved in patients with spina bifida and spinal cord injury. ${ }^{34,35,37}$ By transferring the ventral (motor) root from a reflex arc of a somatic nerve (typically L-5 or S-1) to the ventral root of a nerve root that normally controls autonomic functions (typically S-2, S-3, or S-4), Xiao et al. reported that patients were able to initiate reproducible bladder contractions and voiding by supplying a sensory stimulus in the distribution of the intact dorsal (sensory) root, at the same level as the somatic donor root (Fig. 1) ${ }^{32,34,37}$ Dr. Chuan-Guo Xiao, a practicing urologist in China, has performed this intradural procedure extensively on patients with neurogenic bladder dysfunction related to spinal cord injury and spina bifida. In 2003, Xiao et al. reported that $12(67 \%)$ of 15 adults with spinal cord injury who underwent the Xiao procedure regained "satisfactory bladder control" within 12-18 months of the procedure, associated with a marked reduction in postvoid residual urine and elimination of overflow incontinence and urinary tract infections. ${ }^{35} \mathrm{~A}$ similar study performed by different surgeons in China, using technical variations of the Xiao procedure, reported similarly encouraging results in patients with spinal cord injury. ${ }^{16-18}$
Buoyed by the success of the Xiao procedure in patients with spinal cord injury, Xiao et al. also applied the technique to children with neurogenic bladder dysfunction related to spina bifida. ${ }^{36}$ In 2005 , Xiao et al. reported that $17(85 \%)$ of 20 children with spina bifida who underwent the procedure had "satisfactory bladder control and continence within 8 to 12 months." ${ }^{36}$ Xiao et al. described these improvements in bladder function as "remarkable," noting that all but 1 of the patients who voided voluntarily did not even need to initiate the reflex by scratching themselves in the dermatomal distribution of the donor nerve root to empty their bladders. ${ }^{36}$ As of 2012, Xiao reported that he and his group had performed the Xiao procedure on 1890 patients with spina bifida in China. Of the 506 (27\%) patients available for follow-up, $435(86 \%)$ were reported to have gained significant improvement in bladder control. ${ }^{33}$

Xiao collaborated with the urology group at Beaumont Hospital for the first North American trial of the Xiao procedure in patients with spina bifida. This trial resulted in encouraging but less uniformly beneficial results. ${ }^{22,23} \mathrm{Pe}-$ ters et al. found that 7 (78\%) of 9 patients had a reproducible increase in bladder pressure with stimulation of the donor nerve root dermatome 1 year after surgery. ${ }^{23}$ By 3 years after undergoing the procedure, $7(70 \%)$ of 10 patients who returned for follow-up were considered to be treatment responders, defined as having a voiding efficiency of $>50 \%$, use of CIC less than once a day, stable renal function, and no worsening of motor function..$^{22}$ However, the results published by Peters et al. were not as compelling as those reported by Xiao et al., as all the patients in the Peters et al. study developed transient leg weakness and most patients still leaked urine. ${ }^{22}$

Our multidisciplinary group became aware of the dramatic results claimed for the Xiao procedure approximately 10 years ago as a result of the publication and presentation of the aforementioned studies, and also through our own patients, who had begun requesting that they undergo the procedure based on what they had learned through various media and internet sources. We designed the study we report here to specifically address concerns about the lack of control groups, blinded and unbiased observers, and detailed follow-up, which had been raised in previous publications regarding the Xiao procedure.

We chose to study the Xiao procedure only in patients who were undergoing spinal cord detethering (DT) to minimize the risk to our patients, to allow the performance of a randomized double-blind trial, and to more clearly differentiate the effects of the Xiao procedure from the urological consequences of spinal cord DT. We report our study design, surgical findings, complications, and 


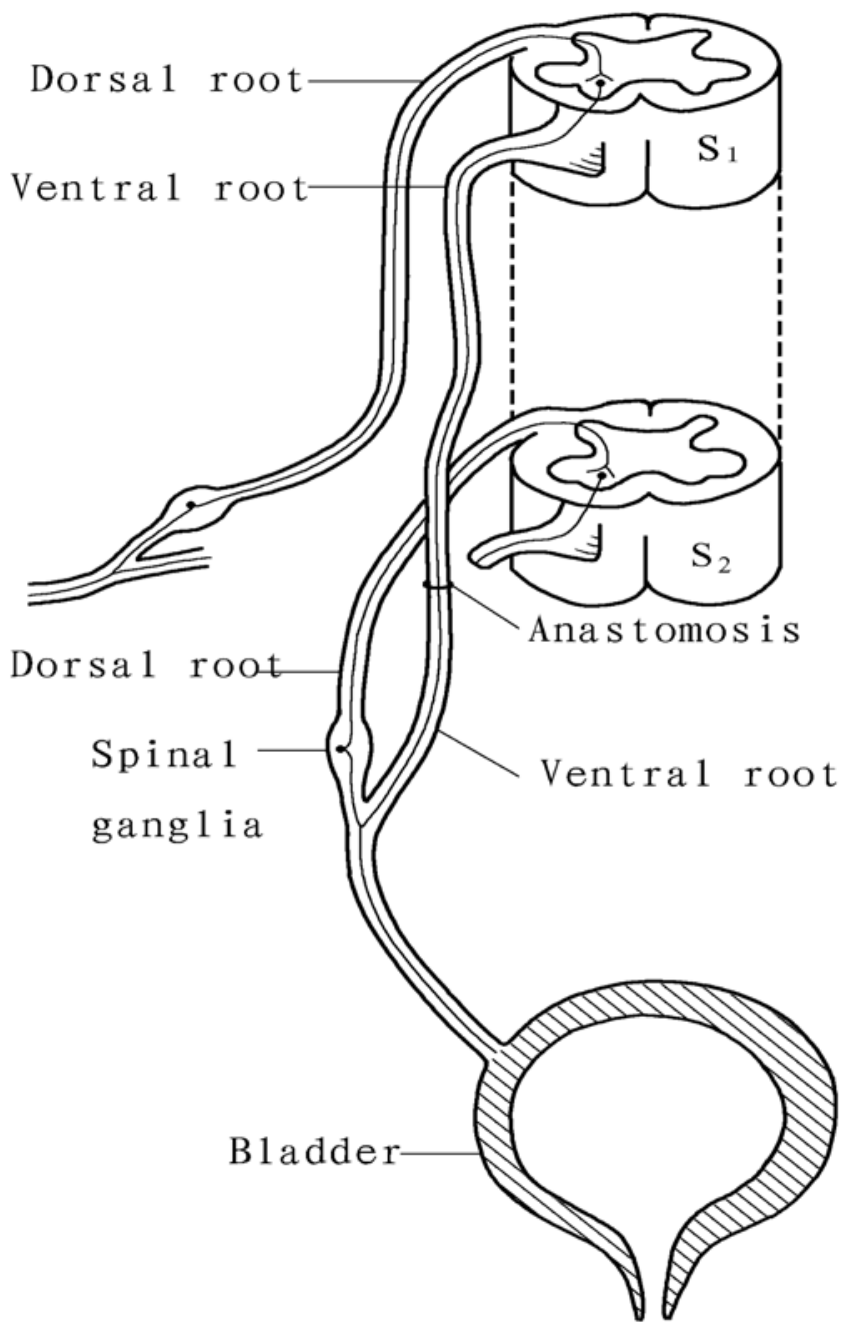

FIG. 1. Diagram of the somatic-to-autonomic intradural anastomosis. The ventral motor root (S-1) is anastomosed to the ventral sacral roots (S-2) to create a skin-CNS-bladder reflex. Reproduced with permission from Lin et al: J Neurosurg Spine 10:452-457, 2009.

outcomes related to voiding, continence, and urodynamic bladder contractions in response to scratching. A separate publication will report QOL measures and more detailed urological and urodynamic outcomes.

\section{Methods}

Members of our research team (L.F.R. and G.F.T.) traveled to China in 2008 to observe Dr. C. G. Xiao and his team perform the Xiao procedure in children with spinal dysraphism. The nuances of patient selection, surgical technique, and postoperative evaluation and management were discussed in the process of designing a protocol at our institution. Approval to conduct the study was obtained from the All Children's Hospital Institutional Review Board in early 2009.

\section{Primary Outcome Measures, Patient Inclusion and Exclusion Criteria, and Preoperative Evaluation}

The primary outcome measure was the ability of chil- dren to void voluntarily and/or in response to cutaneous stimulation. Secondary outcome criteria included muscle strength and sensation, continence, and bladder contractions in response to cutaneous stimulation. Our null hypothesis stated that the addition of the Xiao procedure $(\mathrm{X})$ would not improve these outcome measures in children with myelomeningocele (MM) and lipomyelomeningocele (LMM) undergoing spinal cord DT.

Children younger than 21 years who had neurogenic bladder dysfunction related to MM or LMM, with typical clinical signs and symptoms of tethered cord syndrome, and who required spinal cord DT were eligible for enrollment in the study. Patients and their families were thoroughly educated about the procedure and the study design, with emphasis on the fact that they and their evaluators would be unaware of which procedure they had undergone, DT alone (DT group) or DT plus the Xiao procedure (DT $+\mathrm{X}$ group). There were no important changes to the methods of the study after commencement, except for those mentioned in the text below.

All operations and evaluations were performed by study personnel. Strength was assessed by manual muscle testing $(\mathrm{MMT})^{12,19}$ and ambulation was evaluated using the Pediatric Evaluation of Disability Inventory (PEDI). $\mathrm{CIC}$ and the use of BAMs were terminated 2 weeks prior to preoperative evaluations, and most patients were able to refrain from both of these modalities for the entire 3-year follow-up. The details of these methodological issues are addressed in the online Appendix and will be further explored in future publications.

Patients were enrolled in the study between March 2009 and March 2012, a period of time predetermined at the inception of the study. We chose this time period for practical reasons and because we estimated that a sufficient number of patients could be enrolled within that time period to observe an effect in some patients. Our a priori sample size calculation determined that a minimum sample size of 13 participants would provide $95 \%$ power to detect an increase of $50 \%$ in voiding after dermatome scratching (stimulated urination reflex) for the $\mathrm{DT}+\mathrm{X}$ group compared with the DT group at a 2-sided significance level $(\alpha)$ of $0.05 .{ }^{36}$ A 3 -member independent data safety and monitoring board (DSMB) reviewed patient outcomes semiannually for the first 3 years, then annually for the final 3 years. Our intent was for the DSMB to terminate the study early if there were concerning complications in the experimental group and/or to extend the study period if there were promising results that might require a longer study period.

\section{Description of the Surgical Procedure, Randomization Protocol, and Mechanism for Double Blinding}

Each patient underwent a standard lumbosacral spinal cord DT performed by at least 2 neurosurgeons participating in the study (C.M.C., L.F.R., S.J.G., and/or B.B.S.). If lumbosacral roots were not identifiable at the end of the DT, the patients were excluded from the study. If the lumbosacral nerve roots were present, the patients were assigned to 1 of 2 treatment arms: the control group underwent only the DT procedure (DT group) and the experimental group underwent the Xiao procedure in addition 
to a standard DT procedure (DT+X group). Randomization was achieved when an operating room nurse chose a sealed envelope from a collection of 40 envelopes that had been prepared before the study began. Twenty envelopes assigned the patient to the control group (DT) and 20 to the experimental group (DT+X).

The Xiao anastomosis was performed using the same technique described by Xiao et al. and others in previous studies (Fig. 1). ${ }^{23,33,36}$ Xiao was present in the operating room and supervised the surgery for the first 7 patients enrolled in the study. Under his guidance, we used the same protocols for electromyography (EMG) stimulation, donor and recipient root selection, nerve sectioning, and anastomosis that he had used in China. We also followed his recommended postoperative management instructions, which were later published in a paper in 2012. ${ }^{33}$

Patients who underwent the Xiao anastomosis were informed which donor root was used so that they could subsequently stimulate in the proper dermatomal distribution. Patients in the DT group, in whom the Xiao anastomosis was not performed, were also assigned a donor root based on a randomly chosen side and root, so that they too could scratch their leg in a dermatomal distribution and therefore maintain the double-blinded principles of the experimental design. Patients, their families, and all the evaluators were blinded to which treatment arm each patient was assigned. Further details concerning the surgical procedure, the randomization protocol, and the mechanisms for double blinding can be found in the Appendix.

\section{Evaluations of Voiding, Urodynamic Studies, and Neurological Compromise}

All patients underwent extensive evaluations of voiding in response to cutaneous stimulation, with and without a urodynamic catheter in place, following a strict stimulation protocol that was followed throughout the study. Secondary outcomes of neurological function, mobility, and continence were also evaluated at regular intervals. The methodological details of these evaluations can be found in the Appendix.

\section{Patient and Family Instruction for Cutaneous Stimulation}

All patients in the control (DT) and experimental groups $(\mathrm{DT}+\mathrm{X})$ were instructed to scratch and rub their skin in their assigned donor root sensory distribution whenever they felt the urge to urinate or when they expected to empty their bladder (see Appendix for additional details). Patients and their caregivers were instructed to continue this scratching regimen from the time they were discharged following their surgery all the way through the 3 -year study period.

\section{Timing of Evaluations and Limitations of Reporting}

Spinal MR images and neurosurgical data sheets, which delineated the indications for spinal cord DT, were completed before surgery. Patients were examined 2 weeks after discharge following surgery for evaluation of postoperative complications and to have MMT performed to determine postoperative muscle strength.

The remainder of the evaluations, including evalua- tions of voiding, PEDI questionnaires, and MMT, were performed before surgery and at 3, 6, 9, 12, 18, 24, and 36 months after surgery. Urodynamic studies were performed at the same intervals, with the exception of 3 months after surgery. After careful data review, we have chosen to report the voiding outcome at 12,24 , and 36 months because we found that our results at the other time periods $(3,6,9$, and 18 months) were very similar to those reported in this paper. Muscle strength and sensory function are reported for outcomes at 2 weeks, 6 months, and 12 months.

\section{Discontinued Involvement in the Study}

Patients were excluded from follow-up if they required surgery or developed a condition that would affect the assessment of their bladder function (e.g., bladder augmentation). Patients who required subsequent DT of the spinal cord during the 3-year study period were not eliminated from follow-up unless lumbosacral nerve roots were sectioned or a significant injury occurred to the spinal cord during the subsequent DT operation.

\section{Statistical Methods}

Statistical analysis was performed using SPSS (version 22, IBM Inc.). Descriptive statistics are presented as counts (percentages) for categorical data and mean (standard deviation) or median (range) for continuous data. Comparisons of baseline characteristics and outcomes between groups were performed using the independent $\mathrm{t}$ test or the Mann-Whitney U-test for continuous variables and Fisher's exact test or chi-square test for categorical variables. Comparisons of outcomes within each group from baseline until the end of the study follow-up were performed using the paired t-test or the Wilcoxon signedrank test. All statistical tests were 2-sided, with $\mathrm{p}<0.05$ considered statistically significant.

\section{Results}

\section{Enrollment, Study Duration, and DSMB Termination Decisions}

Twenty of 22 eligible patients scheduled for a spinal cord DT procedure by the neurosurgeons were enrolled in the study between 2009 and 2012. Two patients who were scheduled for spinal cord DT during this time period, and who met all the inclusion and exclusion criteria for the study, declined enrollment because they preferred not to participate in a clinical trial of an experimental procedure (Fig. 2).

The DSMB allowed continued enrollment throughout the entire 3-year study period because the complication rate was not substantially higher in the experimental group. Prolongation of the 3 -year enrollment period was not believed to be justifiable by the DSMB because there were no substantial improvements in the primary and secondary outcome measures in the experimental group that would justify such an extension.

\section{Baseline Demographics and Patient Characteristics}

Patients ranged in age from 1 to 18 years, with a median age of 9 years (Table 1). Thirteen enrollees were boys and 7 were girls. All patients had previously undergone 


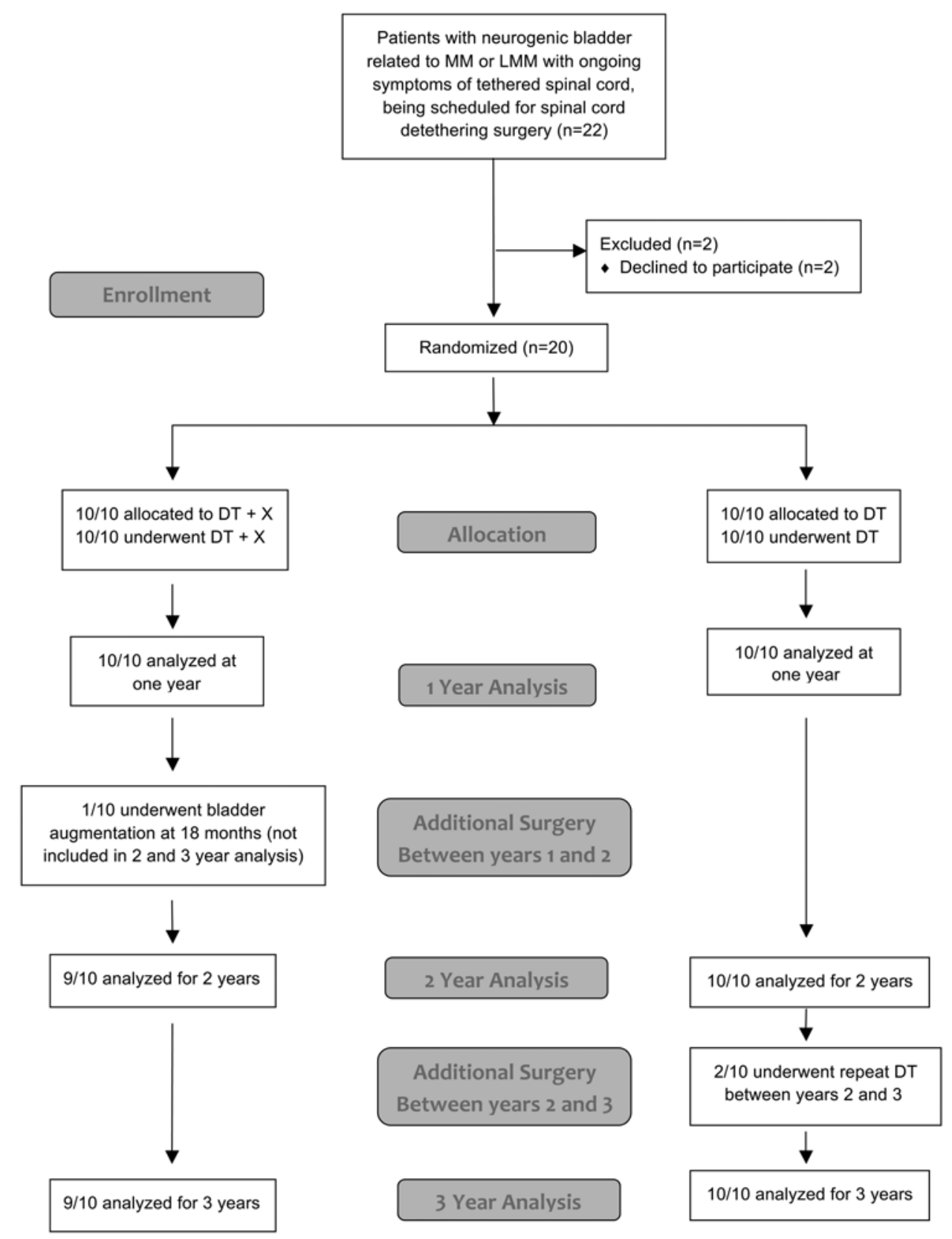

FIG. 2. Consolidated Standards for Reporting of Trials (CONSORT) flow diagram showing the progress of patients through the trial.

spinal cord procedures: 16 had had an MM repaired in utero or shortly after birth, and 4 had undergone DT of a spinal cord lipoma early in life (LMM). In addition to their initial surgery, 9 patients had undergone 1 other DT at some point prior to their enrollment in the study, and 1 patient had undergone 2 previous DTs. Eleven patients had a syrinx present on their preoperative MRI (Table 1). The PEDI caregiver assistance scaled score ranged from 0 to 100 , with a median of 68 . All patients were incontinent of urine, all wore pull-ups or diapers, and none were able to void voluntarily or on command. Further details are available in the Appendix.
Ten participants were randomly assigned to a standard spinal cord DT procedure (DT group), while the other 10 were assigned to the Xiao procedure in addition to a standard spinal cord DT procedure (DT+X group; Fig. 2). There were differences in the baseline characteristics between the groups, but none of the differences were statistically significant (Table 1). Patients were younger (median 6.5 vs 9.5 years), were less likely to have a syrinx ( $40 \%$ vs $70 \%$ ), and were less likely to have undergone multiple DTs (30\% vs 70\%) in the DT+X group than patients in the DT group (Table 1). Patients in the DT $+X$ group were also less likely than patients in the DT group to have back pain 


\begin{tabular}{|c|c|c|c|c|c|c|c|c|c|c|c|c|}
\hline \multirow[b]{2}{*}{$\begin{array}{l}\text { Case } \\
\text { No. }\end{array}$} & \multirow[b]{2}{*}{$\begin{array}{l}\text { Age (yrs), } \\
\text { Sex }\end{array}$} & \multirow[b]{2}{*}{$\begin{array}{c}\text { Type of } \\
\text { SD }\end{array}$} & \multirow[b]{2}{*}{ Level* $^{*}$} & \multirow[b]{2}{*}{$\begin{array}{c}\text { No. of } \\
\text { Previous Ops }\end{array}$} & \multirow[b]{2}{*}{$\begin{array}{l}\text { In Utero } \\
\text { Repair }\end{array}$} & \multirow[b]{2}{*}{$\begin{array}{l}\text { Syrinx } \\
\text { Present }\end{array}$} & \multicolumn{6}{|c|}{ Symptoms of Tethered Cord Prior to Surgery } \\
\hline & & & & & & & $\begin{array}{l}\text { Back } \\
\text { Pain }\end{array}$ & $\begin{array}{l}\text { Leg } \\
\text { Pain }\end{array}$ & $\begin{array}{c}\text { Leg } \\
\text { Weakness }\end{array}$ & $\begin{array}{c}\text { Foot } \\
\text { Deformities }\end{array}$ & $\begin{array}{l}\text { Worsening } \\
\text { Bladder Fxn }\end{array}$ & $\begin{array}{c}\text { Worsening } \\
\text { Scoliosis }\end{array}$ \\
\hline \multicolumn{13}{|l|}{$\mathrm{DT}+\mathrm{X}$} \\
\hline 1 & $5, M$ & MM & $L-3$ & 1 & - & - & - & - & - & - & + & + \\
\hline 2 & $4, \mathrm{~F}$ & MM & $L-4$ & 1 & - & + & - & - & - & + & - & - \\
\hline 3 & $9, \mathrm{M}$ & MM & $L-5$ & 2 & - & + & - & - & - & - & + & - \\
\hline 4 & $1, M$ & MM & $\mathrm{L}-4$ & 1 & - & - & - & - & - & + & + & - \\
\hline 5 & $12, \mathrm{~F}$ & MM & T-12 & 1 & - & - & - & - & - & - & - & + \\
\hline 12 & $7, \mathrm{M}$ & MM & $L-4$ & 1 & + & - & + & - & - & + & - & + \\
\hline 15 & $6, M$ & LMM & $L-5$ & 2 & - & - & - & - & + & + & + & - \\
\hline 16 & $4, F$ & LMM & S-1 & 1 & - & - & - & + & + & + & + & - \\
\hline 17 & $16, F$ & $\mathrm{MM}$ & $\mathrm{L}-4$ & 2 & - & + & + & - & + & - & + & - \\
\hline 19 & $9, \mathrm{M}$ & MM & $\mathrm{L}-4$ & 1 & - & + & + & + & - & - & + & + \\
\hline Total & $6.5 \dagger$ & & & & $10 \%$ & $40 \%$ & $30 \%$ & $20 \%$ & $30 \%$ & $50 \%$ & $70 \%$ & $40 \%$ \\
\hline \multicolumn{13}{|l|}{ DT only } \\
\hline 6 & $9, \mathrm{M}$ & LMM & L-3 & 1 & - & - & - & - & - & - & - & + \\
\hline 7 & $9, \mathrm{M}$ & MM & L-3 & 2 & - & + & + & - & - & + & + & - \\
\hline 8 & $14, \mathrm{M}$ & MM & L-3 & 2 & - & + & + & - & + & - & + & + \\
\hline 9 & $18, \mathrm{M}$ & MM & L-4 & 2 & - & + & + & - & + & - & - & - \\
\hline 10 & $10, M$ & MM & $L-5$ & 2 & - & + & + & - & + & - & + & + \\
\hline 11 & $14, \mathrm{M}$ & MM & L-3 & 3 & - & + & - & - & - & - & - & + \\
\hline 13 & $13, F$ & LMM & S-1 & 2 & - & - & + & + & - & + & - & + \\
\hline 14 & $2, F$ & MM & $L-4$ & 1 & + & - & - & - & - & + & + & - \\
\hline 18 & $1, F$ & MM & $L-5$ & 1 & - & + & - & - & + & + & + & - \\
\hline 20 & $7, M$ & MM & $L-4$ & 2 & - & + & + & + & + & + & + & - \\
\hline Total & $9.5 \dagger$ & & & & $10 \%$ & $70 \%$ & $60 \%$ & $20 \%$ & $50 \%$ & $50 \%$ & $60 \%$ & $50 \%$ \\
\hline
\end{tabular}

Fxn = function; SD = spinal dysraphism; + = yes; - = no.

* Level = neurological level.

$\dagger$ Median age.

(30\% vs 60\%), leg weakness ( $30 \%$ vs $50 \%$ ), and worsening scoliosis (40\% vs 50\%) as an indication for spinal cord DT (Table 1).

\section{Surgical Details and Operative Complications}

All spinal cords were completely detethered and all patients were found to have anatomically intact lumbosacral nerve roots after DT, allowing randomization of all patients who underwent DT. Donor roots were derived from the left side in 7 patients and from the right side in 3; L-5 was used as the donor root in half the patients, $\mathrm{S}-1$ and L-4 each served as donors in 2 patients, and T-11 was used as a donor in 1 additional patient. T-11 was chosen in Patient 5 because we and Dr. Xiao believed that stimulation at lower nerve roots produced only minimal EMG responses. The extent of donor (ventral) root sectioning was $100 \%$ in 3 patients, $90 \%$ in 1 patient, $50 \%$ in 4 patients, $40 \%$ in 1 patient, and 33\% in 1 patient (Table 2; see Appendix for additional details related to the surgical procedure).

The median operative time for all patients was 137 minutes, with a range of 56 to 319 minutes. The median total operating time for the DT+X group was significantly longer than for the DT group (191 vs 106 minutes; $\mathrm{p}=0.024$ ).
The overall median blood loss was $30 \mathrm{ml}$ (range 10-200 $\mathrm{ml}$ ), with no difference between the groups: $27.5 \mathrm{ml}$ (range 20-200 ml) in the DT+X group compared with $50 \mathrm{ml}$ (range 10-200 ml) in the DT group. No wound infections occurred in the DT group, but $50 \%$ of the patients in the $\mathrm{DT}+\mathrm{X}$ group had a wound infection that necessitated oral antibiotics, which was a statistically significant difference $(p=0.03)$. No revision of a lumbar wound was required and no CSF leaks occurred in either group.

\section{Additional Spinal Cord and Bladder Surgery}

Three patients ( 1 in the DT+X group and 2 in the DT group) underwent further spinal cord or bladder surgery during the 3-year follow-up (Fig. 2). In the DT+X group, Patient 5 underwent a bladder augmentation 18 months after her spinal cord surgery due to persistently low bladder volumes, hydronephrosis, and worsening renal function. Because her bladder augmentation affected her urodynamic testing and her responses to questionnaires, she was thereafter excluded from the study. Two patients in the DT group underwent repeat DT procedures during their 3-year follow-up: Patient 14 underwent a repeat DT 23 months later due to worsening tone in the lower extremities and 
TABLE 2. Details of the intradural nerve anastomosis in patients who underwent the Xiao procedure

\begin{tabular}{|c|c|c|c|c|c|c|}
\hline $\begin{array}{l}\text { Case } \\
\text { No. }\end{array}$ & Donor Root (side) & $\%$ Ventral Donor Root & Recipient Roots (side) & Grafts Used & Graft Length (mm) & $\begin{array}{l}\text { No. of Sutures } \\
\text { for Anastomosis }\end{array}$ \\
\hline 1 & $\mathrm{~L}-5(\mathrm{It})$ & 50 & S3-4 (It) & 0 & NA & 1 \\
\hline 2 & $\mathrm{~L}-4$ (It) & 50 & S-3 (It) & 0 & NA & 1 \\
\hline 3 & $L-5$ (It) & 50 & S3-4 (It) & 0 & NA & 1 \\
\hline 4 & $\mathrm{~L}-5(\mathrm{rt})$ & 90 & S3-4 (It) & 0 & NA & 1 \\
\hline 5 & $\mathrm{~T}-11(\mathrm{It})$ & 100 & S3-4 (It) & 0 & NA & 1 \\
\hline 12 & S-1 (It) & 100 & S3-4 (It) & 0 & NA & 1 \\
\hline 15 & $\mathrm{~L}-5(\mathrm{rt})$ & 40 & S3-4 (It) & 0 & NA & 1 \\
\hline 16 & $\mathrm{~L}-5(\mathrm{It})$ & 33 & S3-4 (It) & 1 & 6 & 2 \\
\hline 17 & $\mathrm{~S}-1(\mathrm{rt})$ & 100 & $\mathrm{~S} 2-4(\mathrm{It})$ & 0 & NA & 1 \\
\hline 19 & $\mathrm{~L}-4(\mathrm{It})$ & 50 & $\mathrm{~S}-2(\mathrm{rt})$ & 2 & $7 \& 13$ & 4 \\
\hline
\end{tabular}

worsening syringomyelia, and Patient 18 underwent repeat DT just prior to her 3-year follow-up because of frequent urinary tract infections and a worsening syrinx on MRI. Both patients in the DT group who underwent additional surgery remained in the study because their bladders were not surgically manipulated during the 3-year follow-up, no spinal cord injury or nerve root transection was noted at the time of surgery, and they did not experience a notable decline in neurological function after the surgery (Fig. 2).

\section{Urodynamic Testing}

\section{Voiding in Response to Scratching}

No patient in either group was able to void $(>20 \%$ total bladder capacity [TBC]) in response to systematic scratching of dermatomes by study personnel prior to surgery. During the 3-year follow-up, 2 patients in the DT+X group and 4 in the DT group were able to void $>20 \%$ of their TBC around the urodynamic catheter in response to scratching, at some point during the 3 -year follow-up (Table 3).

In the DT+X group, Patient 1 was able to void $>20 \%$ of his TBC when scratched on the predicted side, outside the dermatomal distribution of the sacrificed ventral root, at years 1 and 2, but he lost that ability by year 3 (Table 3 ). Patient 3 of the DT $+X$ group was able to void in response to scratching in the first year when scratched on the same side of the ventral nerve sacrifice, when scratched within and outside the dermatomal distribution of the sacrificed nerve root. However, he lost that ability at years 2 and 3. The remaining 8 patients in the DT+X group were unable to void in response to scratching at any time during the study.

The 4 patients in the DT group who were able to void in response to scratching by study personnel had results that were similarly inconsistent with those observed in the experimental group. Patient 10 in the DT group, who had a consistently overactive bladder, was able to void $>20 \%$ of TBC during the entire 3-year follow-up period, in response to scratching at the randomly assigned dermatomal and nondermatomal levels. Patients 7, 13, and 18 in the DT group also intermittently voided in response to scratching at various time intervals, but the results were not consistent. No patient in either group was able to void normally, reproducibly, or consistently during urodynamic testing at any point during the study period.

\section{Bladder Contractions in Response to Scratching}

Bladder contractions in response to scratching occurred in patients in both groups, before and after surgery, but there were no statistically significant differences in the frequency of contractions between the groups. Before surgery, $40 \%$ of patients in both groups had a $>10 \mathrm{~cm} \mathrm{H}_{2} \mathrm{O}$ increase in detrusor pressure when scratched somewhere between T-9 and S-1. During the 3-year study period, patients in the DT group tended to have more detrusor contractions in response to scratching than those in the DT+X group at every time interval (Fig. 3; Table 3). Detrusor contractions were not more likely to occur at the designated dermatomal level or predicted side of the body in either group at any time interval (Fig. 3; Table 3). No patient in the DT+X group had reproducible, convincing bladder contractions like those described by Xiao et al. and Peters et al. in previous publications. ${ }^{22,23,36}$

\section{Continence}

No patient in either group was continent of urine before surgery or at any time during follow-up. All patients wore diapers or pull-ups before and at all points in the 3-year follow-up.

\section{Voiding Voluntarily}

No patient in either group could voluntarily void $>20 \%$ of their TBC on their own, before or at any time during the 3-year follow-up, with or without the patient or their caregiver scratching vigorously in the designated dermatomal distribution. In the $\mathrm{DT}+\mathrm{X}$ group, Patient 3 was able to reliably void $<50 \%$ of his TBC if his caregiver poured warm water on his back during his baths, but he could not void if he was scratched in the dermatomal distribution of the ventral nerve root sacrificed for his anastomosis. This reproducible warm-water reflex gradually dissipated after 2 years and it was no longer demonstrable at his 3-year follow-up. Patients in both groups intermittently dribbled urine, but none could initiate a urine stream voluntarily at any point, before surgery or during the 3-year follow-up. 


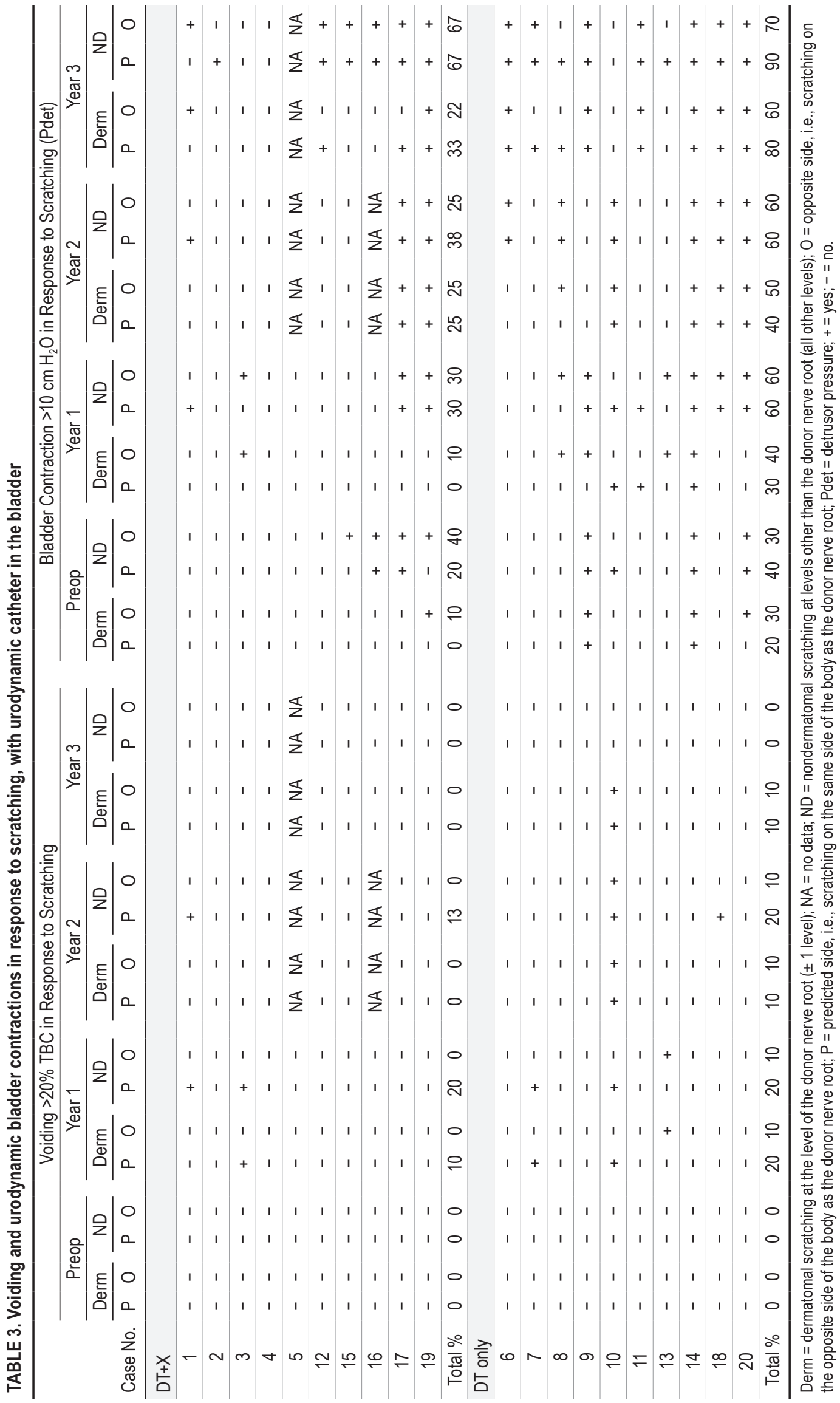




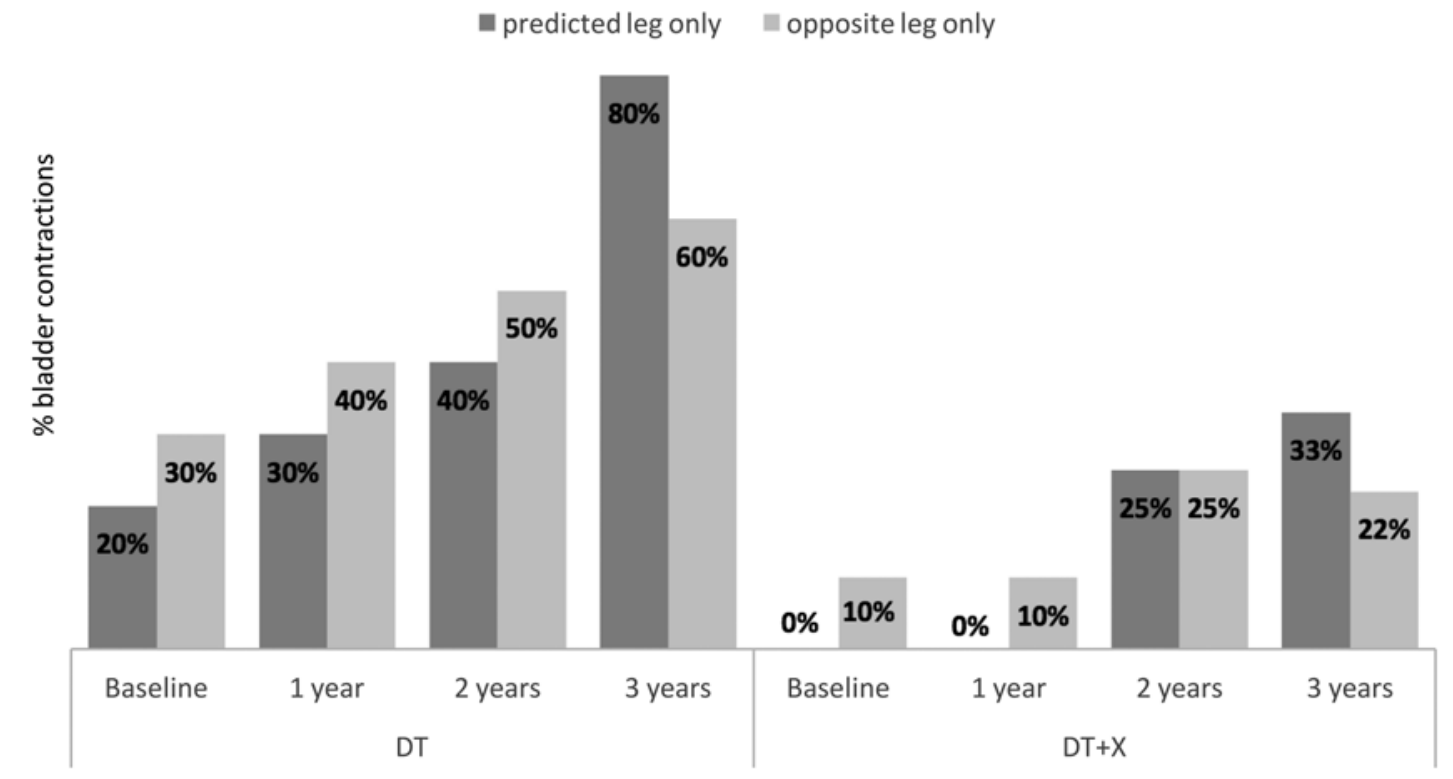

FIG. 3. Bar graph showing frequency of bladder contractions $>10 \mathrm{~cm} \mathrm{H}_{2} \mathrm{O}$ in response to scratching at dermatomal levels ( \pm 1 level) over the 3-year follow-up (see Appendix for details of the scratching protocol). Bladder contractions that resulted from scratching both legs, only the predicted leg, and only the opposite leg were more frequent in the control group (DT) than in the experimental group (DT+X).

\section{Voiding in Response to Scratching and Without Urodynamic Catheter}

Voiding in response to systematic and vigorous scratching by study personnel, with the urodynamic catheter removed, was rarely possible in any patient in either study group at any time interval. Voiding in response to scratching with the catheter removed occurred in the same patients who voided with the urodynamic catheter in place (Table 3), but their voiding was even less frequent with the catheter out. No patient in either group voided normally or reproducibly at any time during the study period, on their own or with study personnel scratching, with or without a urodynamic catheter in place.

In the DT+X group, Patient 1 was the only patient who ever voided without a thin urodynamic catheter in place, at any time interval when study personnel scratched him: he voided once at the 2-year time period when he was scratched on the predicted side and in the dermatomal distribution of the nerve root sacrifice. In the DT group, however, 2 patients were able to void without a catheter in place when study personnel scratched them, but the response occurred only at the 2-year time point. Patient 10 of the DT group, who had a consistently overactive bladder, did void $>20 \%$ of his TBC with the urodynamic catheter out at 2 years when scratched in and outside the designated dermatomal level, on both sides of his body. Patient 18 in the DT group also voided at 2 years when scratched outside her dermatomal level, but on the predicted side based on the arbitrarily assigned dermatome.

\section{Neurological Complications}

One (10\%) of 10 patients in the DT+X group and $2(20 \%)$ of 10 in the DT group experienced a decline in muscle strength at their designated donor root level during their evaluation 2 weeks after surgery (Appendix Table 1). In the patient in the DT $+\mathrm{X}$ group, weakness at the designated root level persisted through 12 months and was most likely attributable to the partial ventral root sacrifice. In the 2 patients in the DT group, new weakness partially resolved and was believed to be related to the spinal cord DT procedure because the loss in strength occurred bilaterally.

Only 1 patient in the study, Patient 13 of the DT group, experienced a decline in ambulatory ability in the first year after surgery (Appendix Table 2). The other patients in both groups experienced similar improvements in the PEDI functional and caregiver assistance mobility scaled scores at 1 year (Appendix Figs. 1 and 2, Appendix Table 2 ). When the 2 groups were compared at 1 year, there were no statistically significant differences in the changes in these measurements. Further details concerning neurological function, ambulatory status, and PEDI scores over the 3 -year time period can be found in the Appendix.

\section{Discussion}

To our knowledge, our study is the first investigation of the Xiao procedure to include a control group, allowing us to more critically analyze the subjective improvements in bladder function and continence that have been the cornerstone of many of the spectacular results reported in previous publications and in medical conferences around the world. By comparing patients in the control (DT) group to those in the experimental (DT+X) group, we attempted to distinguish the effects of DT from those of X ([DT+X] - DT $=X$ ). In our study, patients with MM and LMM who underwent the Xiao procedure during spinal cord DT (the DT+X group) were no more likely to be able to control their urination, to achieve continence, or to have a demonstrable urodynamic bladder contraction in response to 
cutaneous stimulation than patients who only underwent spinal cord DT.

We found the double-blind, randomized methodology used in this study to be essential to the critical evaluation of our results, because patients, their caregivers, and the physicians caring for these patients were all highly vested in the belief that the Xiao procedure might provide patients continence and voluntary voiding. Although the determination of urinary continence was fairly clear and was based on the need for diapers or pull-ups to contain urine, other measures of success were more opaque. For example, patients and their caregivers tended to attribute any voluntary voiding to a functional reflex arc when objective observers were able to clearly determine that the patients were actually utilizing the Credé or Valsalva maneuver to leak or dribble urine. Similarly, hopeful but blinded investigators were quick to attribute a urodynamic bladder contraction in response to cutaneous stimulation to the presence of an intact reflex arc, even if the patient was known to have an overactive bladder that was prone to spontaneous uninhibited contractions. To our knowledge, this is the first evaluation of the Xiao procedure in patients with spinal dysraphism to include patients or evaluators who were blinded to whether the Xiao procedure was performed.

\section{Discrepancies Between Current Results and Previously Published Results}

Our experiences with the Xiao procedure in patients with MM and LMM were similar to those of other investigators in some ways, but our results were different in many important respects. As expected, the addition of the Xiao procedure to spinal cord DT added approximately 90 minutes of additional operating time and resulted in a higher wound infection rate. ${ }^{24}$ Similar to previous investigators, ${ }^{22,23,36}$ we found that a significant number of our patients did have neurological decline after surgery, but we actually found that more patients in the control group (DT) experienced a decline in muscle strength after surgery than those who also underwent the Xiao procedure $(\mathrm{DT}+\mathrm{X}){ }^{33}$ Like Xiao et al. and Peters et al., we found that most of our patients were able to stay off CIC and BAMs for the entirety of the study, without significant hydronephrosis or renal dysfunction, with the exception of 1 patient in the $\mathrm{DT}+\mathrm{X}$ group who required bladder augmentation. ${ }^{22,33,36} \mathrm{~A}$ more detailed analysis of these urological parameters will be reported elsewhere.

Despite the similarities between our experience and the experiences described by other investigators, our key outcome results are entirely different from those published by other investigators. Most importantly, the patients in our study who underwent the Xiao procedure were no more likely than patients in the control group to be continent, to be able to void voluntarily or in response to scratching, or to have a urodynamic bladder contraction in response to scratching. ${ }^{22,23,33,36} \mathrm{Xiao}$ et al. found that $17(85 \%)$ of 20 patients with spina bifida gained "satisfactory bladder control and continence" within 8 to 12 months of surgery, with a demonstrable bladder reflex after cutaneous stimulation during urodynamic studies. ${ }^{36}$ Peters et al. found that $7(78 \%)$ of 9 patients had a reproducible increase in bladder pressure with stimulation at 1 year. ${ }^{23}$ By 3 years, 7 $(70 \%)$ of their 10 patients were considered responders and able to void efficiently, while relying on CIC less than once a day. ${ }^{22}$ Xiao and his team certainly have performed this procedure on the largest number of children worldwide. Based on the nearly 2000 patients with spina bifida who had undergone the procedure by his team as of 2012, Xiao reported a significant improvement in bladder control in approximately $85 \%$ of the 506 patients he was able to evaluate during follow-up. ${ }^{33}$ Even if we ignore the results of our control patients, our results differ dramatically from those previously published.

\section{Limitations of the Study}

Our randomized, double-blind, controlled study design proved advantageous in the objective assessment of the compelling benefits that other investigators have reported with the Xiao procedure, but the lack of controlled voiding or an improvement in continence in our patients requires further scrutiny, particularly in the context of previous publications reporting successful results with this procedure.

During his visit with us at the inception of our study, Dr. Xiao expressed concern about the randomization of patients and the performance of the procedure on patients who were undergoing spinal cord DT. Dr. Xiao was concerned that our patients were undergoing a more extensive spinal cord procedure than he might perform, which might lessen the chance of improvement after the Xiao procedure. However, based on our observation of Xiao and his colleagues performing surgery in China, we believe that spinal cord DT is necessary for the proper completion of the nerve root electrophysiological testing, anatomical identification, and nerve root transfer required to perform the Xiao procedure properly in the typical patient with MM or LMM and neurogenic bladder dysfunction who would be considered for this procedure in the US. In our limited observations, Xiao's team performed an extensive exposure of the spinal cord and cauda equina in children with spinal dysraphism, similar to the exposure during the typical spinal cord DT procedure we routinely perform.

Based on Xiao's 2012 guidelines, it is clear that the typical patient selected for the procedure by Xiao differs from the type of patients with spinal dysraphism we would typically consider for the procedure in our clinical practice in the US. ${ }^{33}$ Xiao states that most Chinese patients who have undergone the procedure are "mobile and do not have a severe form of spina bifida," have "at most one surgery of closure after birth," with "no history of taking anticholinergics."33 Based on our limited observations in China and our conversations with Xiao, he and his team may tend to perform the procedure on patients with the types of spinal dysraphism that are not typically associated with severe neurogenic bladder dysfunction in our practice. The patients enrolled in North American studies by our team and by Peters et al. ${ }^{23}$ did not fit these general guidelines, which may explain some differences in outcome. One of our patients in the experimental group had undergone in utero repair of MM, similar to patients in the study of Peters et al., and Xiao has hypothesized that this procedure may result in less favorable results. ${ }^{33}$ Even with randomization in our 
study, the patients in our 2 study groups had differences in age, the number of previous operations, and the presenting signs and symptoms that might also explain the lack of efficacy of the Xiao procedure in our study.

Despite our strict adherence to Xiao's surgical instruction, there may have been some variations in surgical technique as well. We went to great lengths to minimize the technical variations, including the presence of Xiao during our initial operations as well as collaborations with the urology team at Beaumont Hospital in Michigan, where Xiao served as a surgical assistant to Peters et al. during the first North American trial of this procedure. The operations performed as part of our study were all performed by at least 2 attending pediatric neurosurgeons who had extensive experience with spinal cord DT. Despite these collaborations and the presence of Xiao and the clinical neurophysiologist (William Nantau) involved with the study by Peters et al., ${ }^{23}$ as well as our careful review of Xiao's previous descriptions of nerve root selection, ${ }^{6}$ the EMG and intraoperative nerve root selection protocol remained ambiguous to us.

Starting from S-1 and moving cephalad, we chose the lowest ventral (motor) nerve root that produced a strong EMG response in the leg during intraoperative stimulation of the candidate root, based on the instructions given by Xiao and our collaborators. ${ }^{23}$ We were instructed to use that root, as long as EMG activity was noted in the corresponding muscle group on preoperative testing, regardless of whether muscle strength was present in that nerve root's muscle innervation distribution before surgery. Although we appreciated the necessity of picking the lowest nerve root possible to minimize the need for nerve grafting for a tension-free anastomosis, we were concerned that we were using a donor root that was not innervating a clinically functional muscle group. As a result, while every donor root we sectioned produced a strong EMG response before sectioning, only half of our patients who underwent the Xiao procedure had intact muscle strength at the donor root level before surgery (Appendix Table 1). Similarly, only 6 of the patients who underwent the Xiao procedure had intact sensory function at the level of the donor root prior to surgery (Appendix Table 1).

Even though the use of clinically nonfunctional nerve roots as the donor for the anastomosis appeared counterintuitive to us during the study, excellent results have been reported by Xiao and colleagues after the procedure in neurologically complete patients with paraplegia, in whom a clinically nonfunctional nerve root was also used as the donor root. ${ }^{17,35}$ We find the use of donor nerve roots that are clinically nonfunctional in patients with spinal cord malformations particularly troublesome in light of recent reports by Albright et al., who found that functional activity was limited in nerve roots exiting the spinal cord placode. ${ }^{1,2}$ These inherent physiological inconsistencies have been noted by other authors as well. ${ }^{8,11,26}$

The lack of efficacy of the Xiao procedure performed in our study might also be explained by the difficulties we encountered in sacral nerve root selection. Anatomical identification of the S-2, S-3, and S-4 roots was not difficult after electrical stimulation and mapping of the potential donor nerves, but we found the selection of the specific sacral nerve root unsettling from an electrophysiological standpoint. Because there was very little detail in previous descriptions of the surgical technique provided in clinical papers by Xiao et al. ${ }^{36}$ and Peters et al., ${ }^{23}$ we once again relied on Dr. Xiao's intraoperative instructions. Although we tried to measure bladder contractions in response to stimulation with a urodynamic catheter in the bladder at the time of surgery, we were rarely able to demonstrate such a contraction, in part due to the propensity for urine to leak around the catheter when the patient was in the prone position. Without definite contractions with stimulation, we were instructed to use healthy-appearing sacral roots (preferably S-3) with diameters similar to those of the donor roots. We routinely sectioned the entire sacral root selected, both the dorsal and ventral roots, because there was no way to reliably differentiate the ventral and dorsal sacral rootlets without electrical stimulation data. Even though Xiao et al. and Peters et al. have reported better results than we obtained, neither group has provided detailed information concerning nerve root selection in their previous publications, making it impossible to know if the lack of efficacy in our patients could be explained by surgical variation. ${ }^{23,33,36}$

Similar to patients in the study of Peters et al., 2 of 10 patients in our study population who underwent the Xiao procedure required nerve grafting, a factor that could certainly lessen the chance for successful growth of axons from the donor to the recipient roots. ${ }^{23}$ Xiao's publication of his extensive experience in China does not mention the frequency with which grafts were necessary in his patients. ${ }^{33,36}$

Our study design included systematic, meticulous, and extensive evaluations of bladder contractions occurring in response to cutaneous stimulation during urodynamic studies because other authors have used the presence of this reflex on urodynamic studies as evidence of reinnervation. We do not believe the lack of demonstrable bladder contractions in our patients who underwent the Xiao procedure can be explained by technical limitations of our urodynamic techniques. Contractions were observed in both patient groups, more commonly in the control group. Similarly, the lack of voiding noted in our patients is unlikely the result of inadequate investigation. We tested all patients' voiding multiple times, with the urodynamic catheter in and out, allowing the patients to assume any position they chose to try to initiate voiding. We did not, however, consider a patient to have voided if they pushed on their abdomen or jumped up and down to enable them to empty their bladder. We believe our assessment of voiding and urodynamic contractions was at least as rigorous as other investigators.

\section{Results of the Xiao Procedure Outside of China}

Two Chinese groups, Xiao et al. and Lin et al., have published many articles describing excellent results using the Xiao procedure (or minor variations of the technique) in adults with neurogenic bladder dysfunction related to spinal cord injury.16-18,33,35 Clinicians and patients have been greatly encouraged by the $67 \%-75 \%$ rate of bladder control reported by these investigators, combined with vast improvements in bladder capacity, normalized 
synchronous voiding, and even continence. Unfortunately, similar clinical results have not been reproduced outside of China to our knowledge.

A multidisciplinary group from Denmark embarked on a prospective trial of the effectiveness of the Xiao procedure in adults with spinal cord injury shortly after we began our study. ${ }^{24}$ Similar to our study, they also traveled to China to learn the procedure from Dr. Xiao and then designed a study based on the guidelines they had learned there. In their meticulously reported study, none of the 10 patients with spinal cord injuries who underwent the procedure could initiate voiding, and no changes in bladder pressure were observed after cutaneous stimulation during their 18 -month follow-up. ${ }^{24}$ Disappointed by the lack of a clinically relevant effect in their patients, these researchers questioned the clinical usefulness of the procedure for neurogenic bladder dysfunction. ${ }^{24} \mathrm{~A}$ child with a spinal cord injury who underwent the procedure by our group also failed to show clinical benefit. ${ }^{27}$ While conclusions cannot be drawn based on this single case, our patient's anastomosis was harvested and pathologically examined 2 years after surgery, confirming that very few axons had grown through the anastomotic site..$^{27}$

Confirmatory studies of the effectiveness of the Xiao procedure in patients with spinal dysraphism have been similarly disappointing. Other than the patients described in this report, the only other studies concerning the effectiveness of this procedure when performed outside China have been published by Peters et al. ${ }^{22,23}$ Even though Xiao was a close collaborator with Peters et al., participating in their operations and providing close guidance on clinical management, their results were not nearly as encouraging as those reported by Xiao et al. ${ }^{22,23}$ Xiao considered the 1-year results reported by Peters et al. "premature and misleading," according to his 2012 publication, citing differences in patient selection and the need for nerve grafting in 4 patients. ${ }^{33}$ One year before Peters et al. reported their 3-year results, Xiao stated that Peters' "3-year report showed very satisfactory outcomes that were similar to ours in China." ${ }^{33}$ However, close comparison of the results from Xiao et al. with those from Peters et al. reveals a completely different outcome..$^{22,23,33,36}$ While $17(85 \%)$ of 20 of Xiao's patients "gained satisfactory bladder control and continence" with the ability to initiate voiding spontaneously, only 1 of 10 of Peters' patients was dry (of urine) and only 1 patient could void without a Valsalva maneuver to initiate voiding. ${ }^{22,23,33,36}$ Peters et al. acknowledged that their study did not include a control group and that their results did not replicate those of Xiao, while emphasizing that the definition of success in the US may differ from that in China. ${ }^{22}$ Specifically, he noted that there may be a less stringent definition of success in China, where cases of neurogenic bladder dysfunction are not so readily managed with CIC, BAMs, and augmentation as they are in the US. ${ }^{22}$

Xiao has performed this procedure on a much larger number of patients than all the aforementioned studies combined, including ours. While Xiao's experience is extensive, we find the lack of reproducibility of his results in peer-reviewed publications worthy of consideration..$^{11,14}$, 20,22-24,26,31,32,34,37

\section{Conclusions}

This randomized, prospective, double-blind trial of children with MM and LMM undergoing spinal cord DT failed to result in voluntary or stimulation-induced voiding during the 3-year follow-up evaluation in patients who underwent the Xiao procedure. Patients who underwent the Xiao procedure during spinal cord DT did not experience improved continence, control of urination, or an increased frequency of stimulation-induced bladder contractions compared with patients who underwent only DT. This study, in the context of disappointing results reported in other recent studies of the Xiao procedure, raises doubts about the clinical applicability of this procedure. In our opinion, further basic science and animal studies are necessary before future human trials of the Xiao procedure are performed in the US.

\section{Acknowledgments}

This work was financially supported primarily by a research grant from the All Children's Hospital Foundation. Additional financial support was provided by the AANS/CNS Joint Section on Pediatric Neurosurgery and the AANS/CNS Joint Section on Disorders of the Spine and Peripheral Nerves.

We are grateful to Dr. Xiao for his gracious hospitality and extensive instruction during our visit with him in China. We also greatly appreciate his patient instruction of our staff on the selection criteria, surgical technique, and recommended management of our patients when he worked with us during our initial operations as part of this study in March 2009. Kenneth Peters, MD, and William Nantau from Beaumont Hospital were similarly gracious and inclusive in their collaboration with our group. Stuart Bauer, MD, and Paul Austin, MD, provided tireless guidance on urodynamic methods and research methods. Input from T. S. Park, MD, regarding electrophysiological evaluation and surgical techniques was very helpful as we were learning this new procedure. John Kusek, $\mathrm{PhD}$, from the National Institute of Diabetes and Digestive and Kidney Diseases critically analyzed our experimental design and the preliminary analysis of our results, which proved very valuable. Jay Gould, $\mathrm{PhD}$, performed the sample size estimation when we designed the study, and he provided interim data analyses during the first year of the study.

Unyielding support and guidance from the entire Johns Hopkins All Children's Hospital research team and the All Children's Foundation, particularly from Sandy Wismer, was greatly appreciated and essential to the completion of this study. We are also indebted to the late Devon Truong, PA-C, who was instrumental in the completion of innumerable urodynamic studies. Jackie Frost at Shriner's Hospital Tampa, Andrew Combs, RPA-C, at NewYork-Presbyterian, and Joanie Cacace at Johns Hopkins All Children's Hospital were all tireless in their urodynamic efforts. Michael Reisman, MD, provided methodological guidance and access to rapid and reliable renal studies. Jonathan Ellen, MD, and Sharon Ghazarian, $\mathrm{PhD}$, were instrumental in the development and implementation of our database. Mildred Dones, PT, and Kimberly Carson, PT, diligently and accurately assessed MMT and PEDI outcomes. Most importantly, we thank all the patients and families who participated in this study for their dedication and willingness to enroll in a research study that may benefit others.

\section{References}

1. Albright AL: Distal spinal cord stimulation in infants with myelomeningoceles: preliminary study. J Neurosurg Pediatr 15:220-222, 2015

2. Albright AL, Okechi H: Distal cordectomies as treatment for lumbosacral myelomeningoceles. J Neurosurg Pediatr 13:192-195, 2014 
3. Ali ZS, Heuer GG, Faught RW, Kaneriya SH, Sheikh UA, Syed IS, et al: Upper brachial plexus injury in adults: comparative effectiveness of different repair techniques. J Neurosurg 122:195-201, 2015

4. Austin PF: Symptoms and side effects: delicate balance in the management of patients with neurogenic bladder. J Urol 190:1974-1975, 2013

5. Averbeck MA, Madersbacher H: Follow-up of the neurourological patient: a systematic review. BJU Int 115 (Suppl 6):39-46, 2015

6. Dai CF, Xiao CG: Electrophysiological monitoring and identification of neural roots during somatic-autonomic reflex pathway procedure for neurogenic bladder. Chin $\mathbf{J}$ Traumatol 8:74-76, 2005

7. Feldman AB, Haley SM, Coryell J: Concurrent and construct validity of the Pediatric Evaluation of Disability Inventory. Phys Ther 70:602-610, 1990

8. Fowler CJ, Griffiths D, de Groat WC: The neural control of micturition. Nat Rev Neurosci 9:453-466, 2008

9. Freeman KA, Smith K, Adams E, Mizokawa S, Neville-Jan A: Is continence status associated with quality of life in young children with spina bifida? J Pediatr Rehabil Med 6:215-223, 2013

10. Gatti C, Del Rossi C, Ferrari A, Casolari E, Casadio G, Scire G: Predictors of successful sexual partnering of adults with spina bifida. J Urol 182 (4 Suppl):1911-1916, 2009

11. Gomez-Amaya SM, Barbe MF, de Groat WC, Brown JM, Tuite GF, Corcos J, et al: Neural reconstruction methods of restoring bladder function. Nat Rev Urol 12:100-118, 2015

12. Hislop H, Avers D, Brown M: Daniels and Worthingham's Muscle Testing: Techniques of Manual Examination and Performance Testing, ed 9. St. Louis: Elsevier, 2014

13. Kabra AT, Feustel PJ, Kogan BA: Screening for depression and anxiety in childhood neurogenic bladder dysfunction. $\mathbf{J}$ Pediatr Urol 11:75.e1-75.e7, 2015

14. Kurzrock EA: Editorial comment on "Outcomes to sacral nerve rerouting for spina bifida.” J Urol 184:707-708, 2010

15. Lassmann J, Garibay Gonzalez F, Melchionni JB, Pasquariello PS Jr, Snyder HM III: Sexual function in adult patients with spina bifida and its impact on quality of life. J Urol 178:1611-1614, 2007

16. Lin H, Hou C, Chen A: Reconstructed bladder innervation above the level of spinal cord injury to produce urination by abdomen-to-bladder reflex contractions. J Neurosurg Spine 14:799-802, 2011

17. Lin H, Hou C, Zhen X, Xu Z: Clinical study of reconstructed bladder innervation below the level of spinal cord injury to produce urination by Achilles tendon-to-bladder reflex contractions. J Neurosurg Spine 10:452-457, 2009

18. Lin H, Hou CL, Zhong G, Xie Q, Wang S: Reconstruction of reflex pathways to the atonic bladder after conus medullaris injury: preliminary clinical results. Microsurgery 28:429435, 2008

19. Mahony K, Hunt A, Daley D, Sims S, Adams R: Inter-tester reliability and precision of manual muscle testing and handheld dynamometry in lower limb muscles of children with spina bifida. Phys Occup Ther Pediatr 29:44-59, 2009

20. Park JM: Editorial comment on "Outcomes of lumbar to sacral nerve reorouting for spina bifida.” J Urol 184:708, 2010

21. Patel DP, Elliott SP, Stoffel JT, Brant WO, Hotaling JM, Myers JB: Patient reported outcomes measures in neurogenic bladder and bowel: A systematic review of the current literature. Neurourol Urodyn 35:8-14, 2016

22. Peters KM, Gilmer H, Feber K, Girdler BJ, Nantau W, Trock $\mathrm{G}$, et al: US pilot study of lumbar to sacral nerve rerouting to restore voiding and bowel function in spina bifida: 3 -year experience. Adv Urol 2014:863209, 2014

23. Peters KM, Girdler B, Turzewski C, Trock G, Feber K,
Nantau W, et al: Outcomes of lumbar to sacral nerve rerouting for spina bifida. J Urol 184:702-707, 2010

24. Rasmussen MM, Rawashdeh YF, Clemmensen D, Tankisi H, Fuglsang-Frederiksen A, Krogh K, et al: The artificial somato-autonomic reflex arch does not improve lower urinary tract function in patients with spinal cord lesions. $\mathbf{J}$ Urol 193:598-604, 2015

25. Summers SJ, Elliott S, McAdams S, Oottamasathien S, Brant WO, Presson AP, et al: Urologic problems in spina bifida patients transitioning to adult care. Urology 84:440-444, 2014

26. Thüroff JW: Words of Wisdom. Re: outcomes of lumbar to sacral nerve rerouting for spina bifida. Eur Urol 59:173-175, 2011

27. Tuite GF, Storrs BB, Homsy YL, Gaskill SJ, Polsky EG, Reilly MA, et al: Attempted bladder reinnervation and creation of a scratch reflex for bladder emptying through a somatic-to-autonomic intradural anastomosis. J Neurosurg Pediatr 12:80-86, 2013

28. Veenboer PW, Bosch JL, van Asbeck FW, de Kort LM: Paucity of evidence for urinary tract outcomes in closed spinal dysraphism: a systematic review. BJU Int 112:10091017, 2013

29. von Gontard A, Baeyens D, Van Hoecke E, Warzak WJ, Bachmann C: Psychological and psychiatric issues in urinary and fecal incontinence. J Urol 185:1432-1436, 2011

30. von Linstow ME, Biering-Sørensen I, Liebach A, Lind M, Seitzberg A, Hansen RB, et al: Spina bifida and sexuality. J Rehabil Med 46:891-897, 2014

31. Wang HZ, Li SR, Wen C, Xiao CG, Su BY: Morphological changes of cholinergic nerve fibers in the urinary bladder after establishment of artificial somatic-autonomic reflex arc in rats. Neurosci Bull 23:277-281, 2007

32. Xiao CG: Reinnervation for neurogenic bladder: historic review and introduction of a somatic-autonomic reflex pathway procedure for patients with spinal cord injury or spina bifida. Eur Urol 49:22-29, 2006

33. Xiao CG: Xiao procedure for neurogenic bladder in spinal cord injury and spina bifida. Curr Bladder Dysfunct Rep 7:83-87, 2012

34. Xiao CG, de Groat WC, Godec CJ, Dai C, Xiao Q: "SkinCNS-bladder" reflex pathway for micturition after spinal cord injury and its underlying mechanisms. J Urol 162:936-942, 1999

35. Xiao CG, Du MX, Dai C, Li B, Nitti VW, de Groat WC: An artificial somatic-central nervous system-autonomic reflex pathway for controllable micturition after spinal cord injury: preliminary results in 15 patients. J Urol 170:1237-1241, 2003

36. Xiao CG, Du MX, Li B, Liu Z, Chen M, Chen ZH, et al: An artificial somatic-autonomic reflex pathway procedure for bladder control in children with spina bifida. J Urol 173:2112-2116, 2005

37. Xiao CG, Godec CJ: A possible new reflex pathway for micturition after spinal cord injury. Paraplegia 32:300-307, 1994

\section{Disclosures}

The authors report no conflict of interest concerning the materials or methods used in this study or the findings specified in this paper.

\section{Author Contributions}

Conception and design: Tuite, Polsky, Homsy, Reilly, Carey, Winesett, Rodriguez, Storrs, Gaskill. Acquisition of data: Tuite, Polsky, Homsy, Reilly, Carey, Winesett, Rodriguez, Storrs, Gaskill, Tetreault. Analysis and interpretation of data: Tuite, Polsky, Homsy, Reilly, Carey, Winesett, Tetreault, Martinez, Amankwah. 
Drafting the article: Tuite. Critically revising the article: all authors. Reviewed submitted version of manuscript: all authors. Approved the final version of the manuscript on behalf of all authors: Tuite. Statistical analysis: Tetreault, Martinez, Amankwah. Administrative/technical/material support: Amankwah. Study supervision: Tuite. Interpreted urodynamic studies: Polsky, Homsy. Performed surgery: Carey, Rodriguez, Storrs, Gaskill. Electrophysiological testing: Winesett. Research nurse coordinator: Tetreault.

\section{Supplemental Information}

Previous Presentations

Portions of this work were presented at the 83rd Annual Meeting of the AANS on May 5, 2015, in Washington, DC.

\section{Online-Only Content}

Supplemental material is available with the online version of the article.

Appendix. http://thejns.org/doi/suppl/10.3171/2015.10. PEDS15271.

\section{Correspondence}

Gerald Tuite, Johns Hopkins All Children's Hospital,

Neuroscience Institute, Division of Pediatric Neurosurgery, 601 5th St. S, Ste. 511, St. Petersburg, FL 33701. email: gtuite1@ jhmi.edu. 